\title{
Authentication of Botanical Origin in Herbal Teas by Plastid Noncoding DNA Length Polymorphisms
}

\author{
Ali Tevfik Uncu," Ayse Ozgur Uncu," Anne Frary, and Sami Doganlar* \\ Department of Molecular Biology \& Genetics, Izmir Institute of Technology, Urla, Izmir 35430, Turkey
}

\begin{abstract}
The aim of this study was to develop a DNA barcode assay to authenticate the botanical origin of herbal teas. To reach this aim, we tested the efficiency of a PCR-capillary electrophoresis (PCR-CE) approach on commercial herbal tea samples using two noncoding plastid barcodes, the trnL intron and the intergenic spacer between $\operatorname{trnL}$ and $\operatorname{trnF}$. Barcode DNA length polymorphisms proved successful in authenticating the species origin of herbal teas. We verified the validity of our approach by sequencing species-specific barcode amplicons from herbal tea samples. Moreover, we displayed the utility of PCR-CE assays coupled with sequencing to identify the origin of undeclared plant material in herbal tea samples. The PCR-CE assays proposed in this work can be applied as routine tests for the verification of botanical origin in herbal teas and can be extended to authenticate all types of herbal foodstuffs.
\end{abstract}

KEYWORDS: capillary electrophoresis, DNA barcode, food genomics, traceability

\section{INTRODUCTION}

Herbal infusions, commonly referred to as herbal teas, are among the most widely consumed hot beverages. The popularity of herbal teas is mainly due to their claimed health-related properties and consumers' preference for their specific taste and aroma. Herbal teas are sold in the market mainly as tea bags and ground or fragmented loose products. Because it is difficult to identify the botanical origin of a ground or processed product by its appearance, ${ }^{1}$ herbal products such as teas, spices, and dietary supplements are vulnerable to accidental contamination, deliberate substitution, or admixing with a less commercially valuable herbal species and undeclared fillers. ${ }^{2}$ In addition to the fact that such products violate consumer rights to get value-for-money, herbal tea products that are not true-to-label pose a health threat, as the adulterant herbal species may have serious adverse effects such as toxicity or allergenicity. ${ }^{2,3}$ Thus, herbal tea authenticity is not only an issue of food quality but also an issue of food safety. Therefore, reliable test methods that identify the botanical origin of herbal tea products are required to protect the consumer from fraud and authenticate genuine products.

During the past decade, significant progress was made in the field of food genomics, with several DNA-based methods developed and standardized for authenticating the species or varietal origin of food products. ${ }^{4,5}$ DNA-based tests have significant advantages over commonly used protein and metabolite analyses for the identification of species origin in food commodities. ${ }^{5,6}$ DNA is more thermostable compared to proteins; thus, it is more resistant to processing conditions. In addition, although identical genetic information is present in every cell of an organism, protein expression is mostly tissue specific. Moreover, the degeneracy of the genetic code and the presence of noncoding regions may present a greater number of variable data points for the discrimination of species. ${ }^{6}$ In contrast, climatic conditions, agricultural practices, processing methods, and storage conditions can all influence metabolite composition when analytical methods are used to profile a plant product. Indeed, such factors constitute the major challenge to obtain consistent metabolite profiles from the same plant species. $^{5}$ In addition, DNA-based analyses involve less complicated and labor intensive steps compared to protein and metabolite analyses, and novel DNA sequencing and genotyping platforms are constantly being released that address a variety of genomic research needs. As a result, several successful studies have described the identification of botanical origin in food samples based on DNA polymorphism. Significant examples of such work include, but are not limited to, the detection of allergen nut species in processed foods; ${ }^{7-9}$ detection of soft wheat adulteration in spelt flour ${ }^{10}$ and durum wheat-based products; ${ }^{11}$ detection of plant adulterants in saffron; ${ }^{12}$ and identification of the floral composition of and bacterial and fungal contamination in honey. ${ }^{13}$ Investigation of the authenticity of herbal dietary supplements ${ }^{2,14,15}$ and herbal teas ${ }^{1}$ using DNA-barcode analyses produced results that emphasize the need to authenticate the botanical origin of herbal products.

DNA-barcodes that are optimal for verifying botanical origin and detecting the presence of undeclared plant species in a given herbal sample should have certain properties. First, conserved flanking sequences should be present to allow universality, which means that the barcode region should be robustly amplified from a diverse range of taxonomic units. However, the sequence between the conserved primer binding sites should be sufficiently variable to enable the discrimination of different plant species. ${ }^{16}$ Thus, a fine balance between the level of sequence conservation and divergence should be present. For example, one of the widely suggested chloroplastic barcodes, the intron maturase gene $m a t K$, represents one of the most rapidly evolving coding sequences in the plastid genome,

Received: March 10, 2015

Revised: June 4, 2015

Accepted: June 9, 2015

Published: June 9, 2015 
providing a relatively high resolution for plant species discrimination. ${ }^{17}$ However, the high level of sequence divergence is at the expense of sites conserved enough to standardize universal primers. In contrast, another widely utilized chloroplastic barcode, $r b c L$ (large subunit of the ribulose-1,5-bisphosphate carboxylase/oxygenase gene), enables robust PCR amplification due to a high level of sequence conservation, but provides relatively poor resolution for discriminating plants at low taxonomic levels. ${ }^{17,18}$ As a result, no single genomic region has yet been identified as the ultimate barcode, and it is often recommended that barcode regions be used in combination to meet specific research needs. ${ }^{19,20}$

In the present work, two noncoding regions from the chloroplast genome, the trnL (UAA) intron and the intergenic spacer between the trnL (UAA) $3^{\prime}$ exon and trnF (GAA) genes, ${ }^{21}$ were used as DNA-barcodes for the discrimination of herbal species. Primers that anneal at conserved flanking coding regions and amplify variable noncoding sequences have long been available for these two barcode regions, and their universality has been shown with successful PCR amplifications from a wide range of taxonomic groups. ${ }^{21,22,16}$ The rate of insertions/deletions (indels) in the two noncoding regions is at least as high as that of nucleotide substitutions. ${ }^{22}$ The accumulation of indels is reflected by amplified fragment length polymorphisms that enable the discrimination of species based on size separation of the barcode amplicons. Such a discrimination capacity is remarkably useful in food authenticity analyses, because in the presence of multiple species in a food commodity, sequence analyses may fail to produce clean, reliable peaks from a mixture of divergent amplicons. ${ }^{17}$ The length polymorphisms in the trnL (UAA) intron region of the chloroplast genome have been utilized for botanical origin authentication in food products in several studies. James and Schmidt ${ }^{23}$ were successful in developing a simple, PCR-based assay that detects the presence of potential allergen food crops based on trnL (UAA) intron length polymorphisms. By taking advantage of the high fragment size resolution feature of capillary electrophoresis systems, Spaniolas et al. ${ }^{24,25}$ displayed the potential of the trnL (UAA) intron length polymorphisms to identify the botanical origin of plant oils. When highresolution melting (HRM) analysis was employed as the genotyping strategy, melting curves generated by the trnL (UAA) intron amplicons were sufficient to discriminate 11 nut species and detect hazelnut contamination in food products. ${ }^{9}$ HRM analysis of the trnL (UAA) intron amplicons provided sufficient resolution to discriminate 13 legume species and allowed the detection of soybean (Glycine max)-lupine (Lupinus spp.) admixtures. ${ }^{26}$ In addition, trnL (UAA) intron barcode-HRM analysis was successful in authenticating the Protected Designation of Origin (PDO) of legume species Lathryus cleymenum. ${ }^{27}$ Utilization of a similar approach for species discrimination was also demonstrated in fruit juices. ${ }^{28}$ With the present work, we propose a fast and reliable PCRcapillary electrophoresis (PCR-CE) barcode assay that involves the use of two plastid noncoding regions and test its efficiency to authenticate botanical origin on a set of 26 commercial herbal tea products that represent eight species that are common in herbal teas.

\section{MATERIALS AND METHODS}

Herbal Tea Samples and Reference Tissue. The herbal tea samples used in this work included 26 commercial herbal tea products in the form of tea bags. The sample set represented seven types of herbal teas commonly found in retail stores, including three singleingredient products (sage tea, chamomile tea, and linden tea) and four blends of two herbal species (rose hip-hibiscus tea, peppermintlinden tea, peppermint-fennel tea, and anise-fennel tea). Each type of herbal tea was represented by four different commercial products from four different companies, except for peppermint-linden tea, which was represented by products from two companies. Herbal tea samples were purchased from retail stores. Because the aim of this study was to evaluate the performance of the plastid DNA-barcodes and not the herbal tea brands, company names are not provided. Samples of each herbal tea type were randomly assigned numbers (samples 1-4) regardless of the brand names.

Reference tissue samples of sage (Salvia officinalis L.), chamomile (Matricaria chamomilla L.), linden (Tilia cordata Mill.), rose hip (Rosa canina L.), hibiscus (Hibiscus sabdariffa L.), peppermint (Mentha piperita L.), fennel (Foeniculum vulgare Mill.), and anise (Pimpinella anisum L.) were obtained from Aegean University Botanical Garden and Herbarium Research and Application Center (Izmir, Turkey).

DNA Isolation. All DNA isolations were performed using a NucleoSpin Plant II Kit (Macherey Nagel, Duren, Germany) according to the manufacturer's instructions. For sample preparation, silica-dried tissue samples of reference herbal species were ground using a mortar and pestle, and $100 \mathrm{mg}$ of ground tissue was used to isolate DNA. In-house admixtures of two, three, and four herbal species, prepared by mixing ground tissue from relevant reference samples, were also used for DNA extraction. Four different admixtures of pairs of herbal species (linden:peppermint; sage:anise; rose hip:chamomile; sage:linden) were prepared in two different admixing ratios of $95: 5 \%$ and $99: 1 \%$. Admixtures of three (sage:anise:chamomile and linden:peppermint:chamomile) and four (chamomile:peppermin$t$ :anise:linden) herbal species were also prepared by mixing equal weights of ground reference tissue from all participant species. In the case of commercial products, the contents of multiple tea bags from each package were combined prior to sampling $100 \mathrm{mg}$ of dried tissue. All DNA extractions (from reference tissue and commercial samples) were performed in two replicates. The concentration of DNA was measured using a Qubit 2.0 Fluorometer (Life Technologies, Thermo Fisher Scientific Inc., Waltham, MA, USA) with a dsDNA BR Assay Kit (Life Technologies). DNA integrity was checked on a $1.5 \%$ agarose gel.

PCR-CE Barcode Analysis. Primers that amplify the plastid trnL (UAA) intron (primers $c$ and $d$ ) and the intergenic spacer between $\operatorname{trnL}$ (UAA) $3^{\prime}$ exon and $\operatorname{trn} F$ (GAA) gene (primers e and $\mathrm{f}$ ) were introduced by Taberlet et al. ${ }^{21}$ Primer sequences from the $5^{\prime}$ to $3^{\prime}$ direction are as follows: primer c, CGAAATCGGTAGACGCTACG; primer d, GGGGATAGAGGGACTTGAAC; primer e, GGTTCAAGTCCCTCTATCCC; and primer f, ATTTGAACTGGTGACACGAG. For simplicity, the two plastid barcodes are here named after the amplification primers as plastid-cd (Pl-cd) and plastid-ef (Pl-ef). Barcode regions were amplified in $20 \mu \mathrm{L}$ reaction mixtures containing $1 \times$ Colorless GoTaq Flexi PCR buffer, $1.5 \mathrm{mM} \mathrm{MgCl}_{2}, 0.25 \mathrm{mM}$ of each deoxyribonucleotide triphosphate (dNTP) (Promega Corp., Madison, WI, USA), 1 U of GoTaq G2 Flexi DNA Polymerase (Promega Corp.), $0.25 \mu \mathrm{M}$ of each primer, and $5 \mathrm{ng}$ of template DNA. Thermal cycling conditions consisted of one cycle of initial denaturation for $10 \mathrm{~min}$ at $94{ }^{\circ} \mathrm{C}$, followed by 35 cycles of $94{ }^{\circ} \mathrm{C}$ for $30 \mathrm{~s}, 62{ }^{\circ} \mathrm{C}$ for $30 \mathrm{~s}$, and $72{ }^{\circ} \mathrm{C}$ for $60 \mathrm{~s}$, with a final extension step of $10 \mathrm{~min}$ at $72^{\circ} \mathrm{C}$. PCR mixtures with an alternative DNA polymerase [Q5 High-Fidelity DNA Polymerase (New England Biolabs Inc., Ipswich, MA, USA)] were prepared in a volume of $25 \mu \mathrm{L}$ and contained $1 \times$ Q5 Reaction Buffer, $0.2 \mathrm{mM}$ of each deoxyribonucleotide triphosphate (dNTP) (Promega Corp.), 0.5 U of Q5 HighFidelity DNA Polymerase (New England Biolabs Inc.), $0.50 \mu \mathrm{M}$ of each primer, and $5 \mathrm{ng}$ of template DNA. Thermal cycling conditions consisted of one cycle of initial denaturation for $30 \mathrm{~s}$ at $98{ }^{\circ} \mathrm{C}$, followed by 35 cycles of $98{ }^{\circ} \mathrm{C}$ for $10 \mathrm{~s}, 62^{\circ} \mathrm{C}$ for $20 \mathrm{~s}$, and $72{ }^{\circ} \mathrm{C}$ for $30 \mathrm{~s}$, with a final extension step of $2 \mathrm{~min}$ at $72{ }^{\circ} \mathrm{C}$. Barcode amplicons in the 96-well microtiter plates used for PCR amplification were directly loaded on a Qiaxcel Advanced capillary electrophoresis system (Qiagen, Valencia, CA, USA). No postamplification treatment was 
Table 1. Barcode Amplicon Sizes of the Eight Herbal Species Used in This Study

\begin{tabular}{lcc}
\multicolumn{1}{c}{ herbal species } & barcode Pl-cd $[\operatorname{trnL}(\mathrm{UAA})$ intron $]$ & barcode Pl-ef (trnL-trnF intergenic spacer) \\
Foeniculum vulgare & $340 \mathrm{bp}$ and $582 \mathrm{bp}$ & $434 \mathrm{bp}$ \\
Hibiscus sabdariffa & $573 \mathrm{bp}$ & $260 \mathrm{bp}$ and $469 \mathrm{bp}$ \\
Matricaria chamomilla & $488 \mathrm{bp}$ & $442 \mathrm{bp}$ \\
Mentha piperita & $580 \mathrm{bp}$ & $368 \mathrm{bp}$ \\
Pimpinella anisum & $602 \mathrm{bp}$ & $432 \mathrm{bp}$ \\
Rosa canina & $614 \mathrm{bp}$ & $472 \mathrm{bp}$ \\
Salvia officinalis & $558 \mathrm{bp}$ & $370 \mathrm{bp}$ \\
Tilia cordata & $628 \mathrm{bp}$ & $459 \mathrm{bp}$ \\
\hline
\end{tabular}

applied to the samples. The sample loading procedure did not involve a post-PCR labeling step, as the capillary cartridges provided with Qiaxcel DNA High Resolution Kits (Qiagen) are ready-to-use and filled with the staining dye-gel mixture. QX DNA Size Marker 50$800 \mathrm{bp}, \mathrm{v} 2.0$ (Qiagen) was used as the size standard, and QX Alignment Marker $15 \mathrm{bp} / 1000 \mathrm{bp}$ (Qiagen) was used for aligning the size standard fragments. The high-resolution run method OM800 was applied with a sample injection time of $10 \mathrm{~s}$. QIAxcel ScreenGel Software (Qiagen) was used for visualization of the barcode profiles. PCR-CE barcode assays were performed in three replicates for each DNA sample, and capillary electrophoresis runs were performed in two replicates.

Barcode DNA Sequencing. Primers that amplify the two barcodes, $\mathrm{Pl}-\mathrm{cd}$ and $\mathrm{Pl}$-ef, were used for dye-terminator sequencing. Sequencing targets were amplified in $20 \mu \mathrm{L}$ reaction mixtures containing $1 \times$ Colorless GoTaq Flexi PCR buffer, $1.5 \mathrm{mM} \mathrm{MgCl}_{2}$, $0.25 \mathrm{mM}$ of each deoxyribonucleotide triphosphate (dNTP) (Promega Corp.), 1 U of GoTaq G2 Flexi DNA Polymerase (Promega Corp.), $0.25 \mu \mathrm{M}$ of each primer, and $5 \mathrm{ng}$ of template DNA. Thermal cycling conditions consisted of one cycle of initial denaturation for $10 \mathrm{~min}$ at $94{ }^{\circ} \mathrm{C}$, followed by 35 cycles of $94{ }^{\circ} \mathrm{C}$ for $30 \mathrm{~s}, 62{ }^{\circ} \mathrm{C}$ for $30 \mathrm{~s}$, and 72 ${ }^{\circ} \mathrm{C}$ for $45 \mathrm{~s}$, with a final extension step of $10 \mathrm{~min}$ at $72{ }^{\circ} \mathrm{C}$. For each reference/herbal tea sample, PCR reactions were performed in five replicates and combined during the purification step with the DNA Clean \& Concentrator-5 Kit (Zymo Research, Irvine, CA, USA). Purified PCR products were used as template in dye-terminator sequencing reactions, prepared using GenomeLab DTCS Quick Start Kit (Beckman Coulter, Brea, CA, USA) according to the manufacturer's instructions. Sequencing reaction thermal cycling conditions were 30 cycles of $96{ }^{\circ} \mathrm{C}$ for $20 \mathrm{~s}, 50{ }^{\circ} \mathrm{C}$ for $20 \mathrm{~s}$, and 60 ${ }^{\circ} \mathrm{C}$ for $4 \mathrm{~min}$. The reaction mixture for each amplicon was then purified using ZR DNA Sequencing Clean-up Kit (Zymo Research), resuspended in $30 \mu \mathrm{L}$ of sample loading solution (Beckman Coulter), and run on a Beckman CEQ8800 capillary electrophoresis device using the LFR-b method (injection voltage $2.0 \mathrm{kV}$ for $10-15 \mathrm{~s}$, separation temperature of $57^{\circ} \mathrm{C}$, separation voltage of $6.0 \mathrm{kV}$, separation time of $60 \mathrm{~min})$. Sequence peaks were visualized with the "Sequencing" module of Beckman CEQ8800 software version 8.0 (Beckman Coulter). Alignment and assembly of sequences from forward and reverse directions and alignment of reference and sample sequences were performed using the "Investigator" module of the CEQ 8800 (Beckman Coulter) software. The MEGA V6.0 computer program ${ }^{29}$ was used for cross-species sequence alignments with the ClustalW method. Homology search in GenBank Nucleotide Collection was carried out using "nucleotide blast" as the BLAST program with the "megablast" option optimized for identifying highly similar sequences. BankIt sequence submission tool (http://www.ncbi.nlm.nih.gov/ $\mathrm{WebSub} /$ ?form=history\&tool=genbank) was used for sequence submission to the GenBank database.

\section{RESULTS}

Identification of Species Origin in Herbal Tea Products Using the PCR-CE Barcode Assays. The two plastid barcodes (barcode Pl-cd and barcode Pl-ef) were amplified using the DNA from the 8 reference samples and 26 commercial herbal tea products. Barcode amplicons were directly loaded on the capillary electrophoresis system without any post-PCR treatment. Because amplicons from the reference samples constituted the standards in the analysis, they were run simultaneously with the amplicons from the commercial tea samples. Sizes of the barcode amplicons are given in Table 1. A single fragment amplification pattern was observed for all of the species except for fennel and hibiscus, which yielded double bands for barcodes Pl-cd and Pl-ef, respectively. All eight herbal species displayed distinctive fragments with no two species yielding fragments of identical size for either of the two barcodes. The amplicon size of barcode Pl-cd ranged between 340 and $614 \mathrm{bp}$, whereas barcode Pl-ef amplicons ranged between 260 and $472 \mathrm{bp}$ for the eight species. For barcode Pl$\mathrm{cd}$, the minimum fragment size difference $(2 \mathrm{bp})$ was observed between fennel and peppermint, which produced fragments of 582 and $580 \mathrm{bp}$, respectively. However, fennel also displayed an additional $340 \mathrm{bp}$ fragment in its barcode Pl-cd profile. For barcode Pl-ef, the minimum fragment size difference was $2 \mathrm{bp}$ and was observed for fennel (434 bp) and anise (432 bp) and also sage (370 bp) and peppermint (368 bp) (Table 1). Similarly, rose hip and hibiscus produced barcode Pl-ef fragments of similar, but not identical, size (472 and $469 \mathrm{bp}$ ), with the latter yielding an additional band of $260 \mathrm{bp}$ in its profile.

For the sage reference sample, the fragment sizes of the two barcodes were 558 and 370 bp for barcodes Pl-cd and Pl-ef, respectively (Table 1). Barcode sizes of all four commercial sage tea samples matched those of the standard reference (Figure 1), and no additional, unexpected fragments were observed for any of the samples in any of the replicate experiments. Therefore, we concluded that the botanical origins of the tested commercial sage tea products were true-to-label.

The chamomile reference sample gave Barcode $\mathrm{Pl}-\mathrm{cd}$ and $\mathrm{Pl}$ ef fragment sizes of 488 and $442 \mathrm{bp}$, respectively (Table 1 ). Barcode Pl-cd and Barcode Pl-ef profiles of three commercial chamomile tea samples (Figure 2, panels II-IV) perfectly matched with those of the reference sample, proving their identity as authentic chamomile tea. However, sample 4 (Figure 2 , panel V) produced profiles that were inconsistent with the reference for both barcodes. In addition to the single $488 \mathrm{bp}$ band expected for chamomile tea, sample 4 yielded an additional band of $564 \mathrm{bp}$ length for barcode Pl-cd (Figure $2 \mathrm{~A}$, panel V). The barcode Pl-ef assay also produced an extra 359 bp band from sample 4, which was not expected for chamomile (Figure 2B, panel V). Similar results were obtained in all three replicate experiments with the two barcodes. Therefore, results of both barcode assays were in agreement and implied that sample 4 was either adulterated or contaminated with a plant species other than chamomile.

For the linden reference sample, the barcode Pl-cd and Pl-ef fragment lengths were 628 and $459 \mathrm{bp}$, respectively (Table 1). 


\section{Sage Tea}

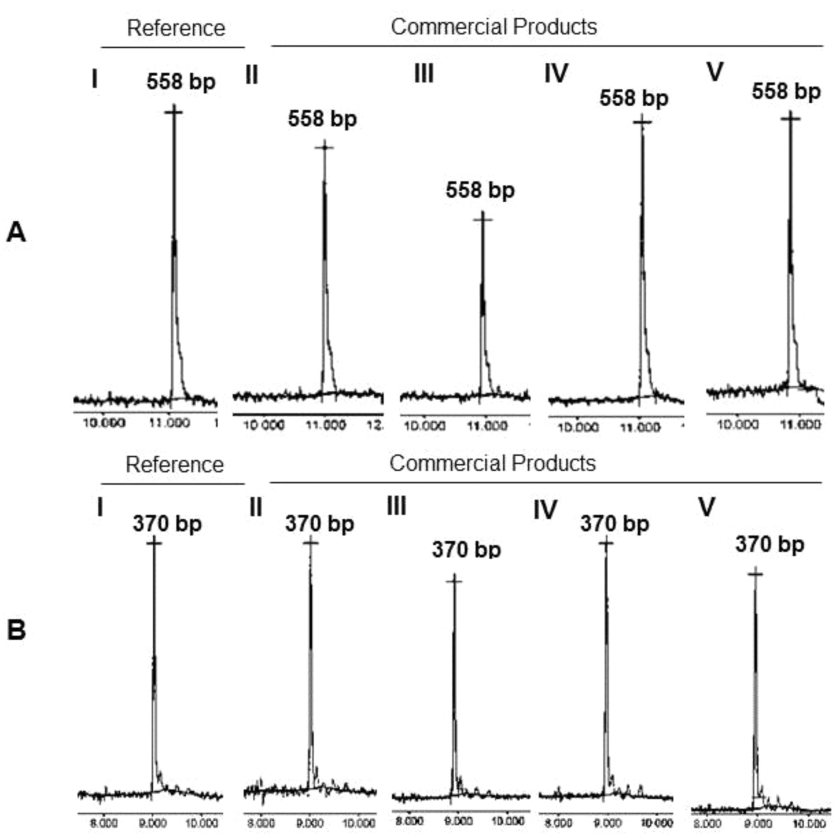

Figure 1. Capillary electropherograms displaying the results of the PCR-CE barcode assays applied on sage tea samples: (A) barcode Plcd assay; (B) barcode Pl-ef assay. Sample order is the same for rows A and B: I, sage reference sample; II, sage tea sample 1; III, sage tea sample 2; IV, sage tea sample 3; V, sage tea sample 4 .

PCR-CE barcode analysis results of the reference and the four commercial linden flower tea samples were in concordance in all three replicate experiments, identifying the commercial samples as authentic linden tea (Figure 3). Peppermint-linden
Linden Tea

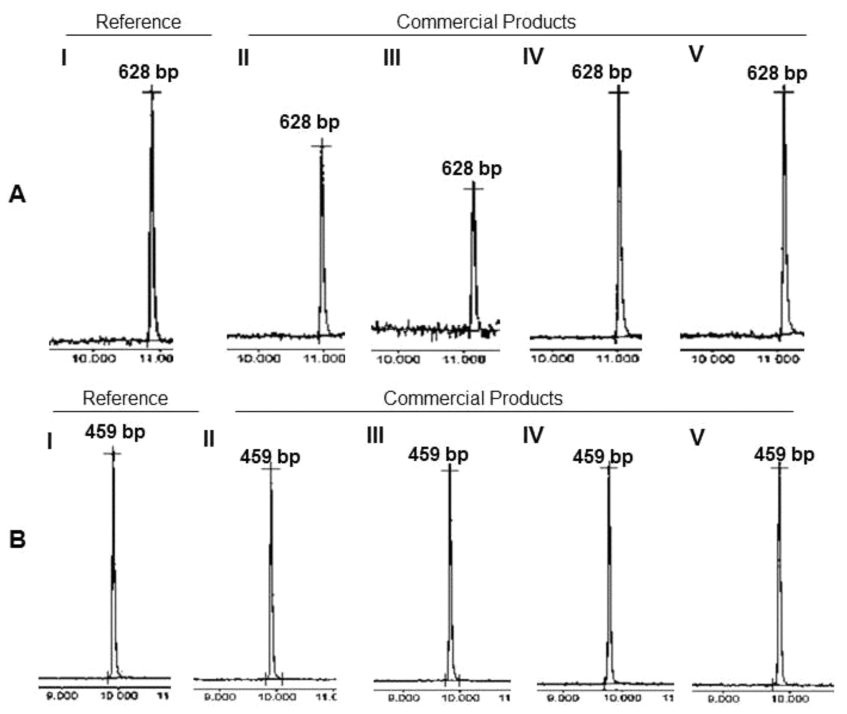

Figure 3. Capillary electropherograms displaying the results of the PCR-CE barcode assays applied on linden tea samples: (A) barcode Pl-cd assay; (B) barcode Pl-ef assay. Sample order is the same for rows A and B: I, linden reference sample; II, linden flower tea sample 1; III, linden flower tea sample 2; IV, linden flower tea sample 3; V, linden flower tea sample 4 .

blends were also examined. According to the product labels, the ratio of peppermint in peppermint-linden blends was $20 \%$ for sample 1 and $30 \%$ in sample 2 . The peppermint reference amplicons were 580 and 368 bp for barcodes Pl-cd and Pl-ef, respectively (Table 1 ). The two peppermint-linden commercial samples were successfully resolved by both barcodes with each barcode electropherogram displaying double bands of

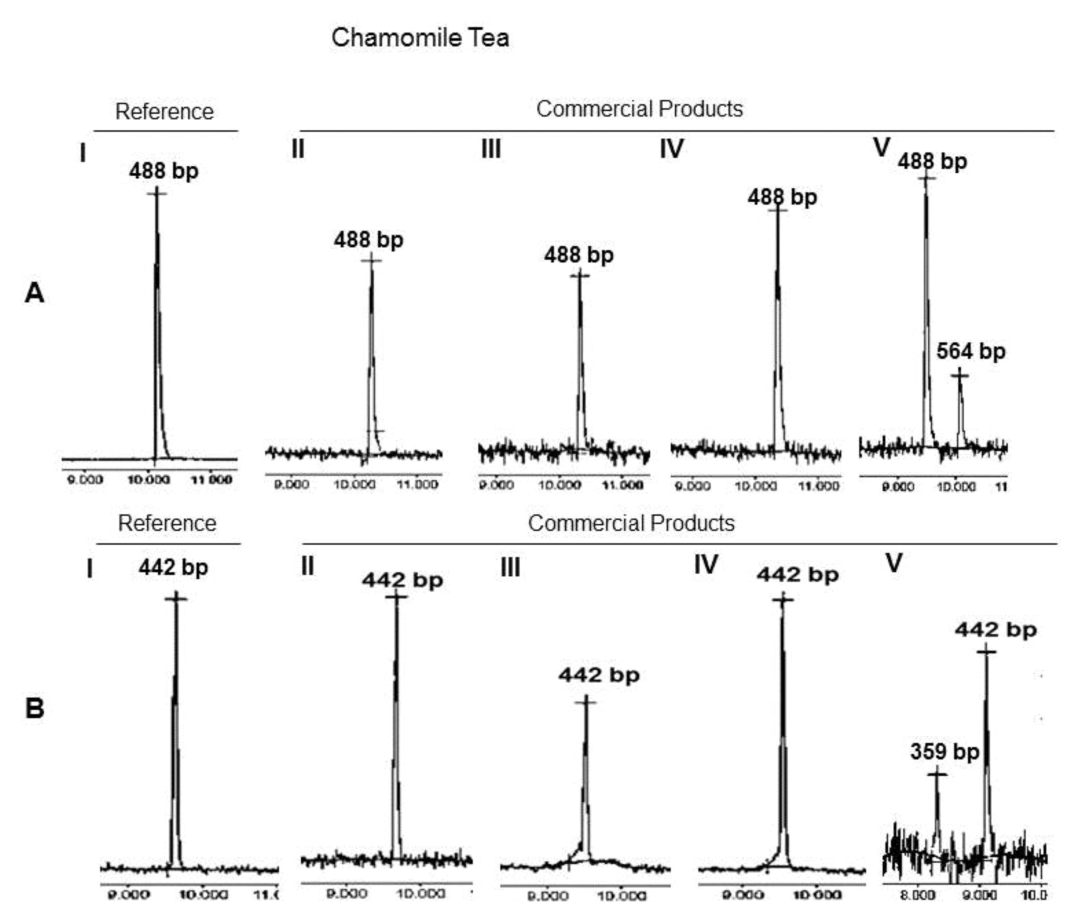

Figure 2. Capillary electropherograms displaying the results of the PCR-CE barcode assays applied on chamomile tea samples: (A) barcode Pl-cd assay; (B) barcode Pl-ef assay. Sample order is the same for rows A and B: I, chamomile reference sample; II, chamomile tea sample 1; III, chamomile tea sample 2; IV, chamomile tea sample 3; V, chamomile tea sample 4. 
sizes identical to the references (Figure 4). In addition, no unexpected band other than the two species-specific fragments

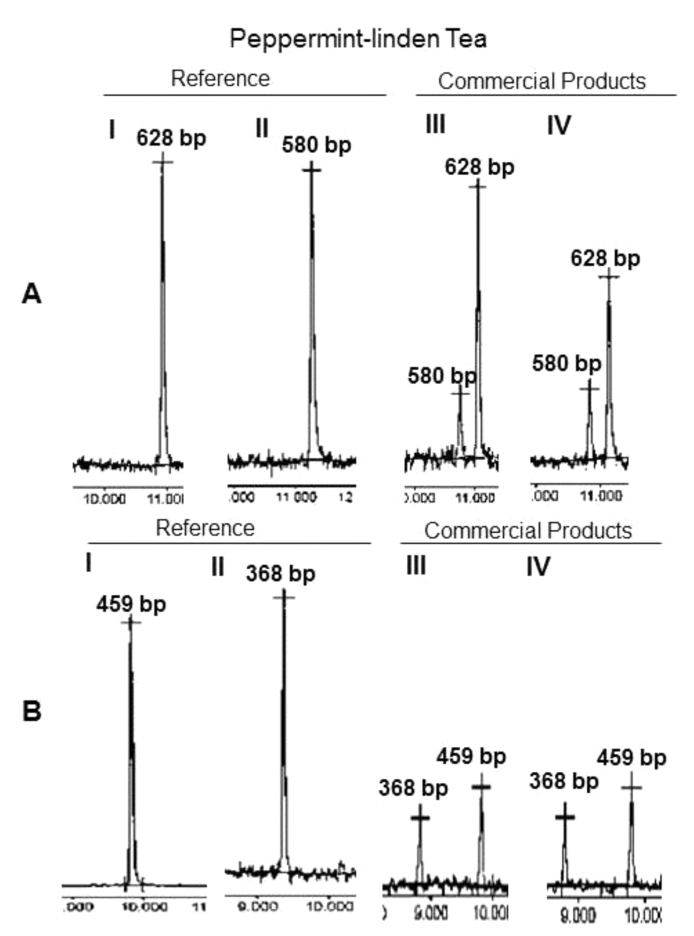

Figure 4. Capillary electropherograms displaying the results of the PCR-CE barcode assays applied on peppermint-linden tea samples: (A) barcode Pl-cd assay; (B) barcode Pl-ef assay. Sample order is the same for rows A and B: I, linden reference sample; II, peppermint reference sample; III, peppermint-linden tea sample 1; IV, peppermint-linden tea sample 2 . was observed in any of the replicate experiments with the two barcodes; therefore, the botanical origin of the two commercial products was identified as a blend of peppermint and linden as indicated on the labels.

All four rose hip tea samples included in this work were blends of rose hip and hibiscus. The ratios of rose hip:hibiscus blends declared on the product labels were $70: 30 \%$ for samples 1 and 3 and 60:40\% for samples 2 and 4 . Rose hip and hibiscus reference samples yielded barcode Pl-cd fragments of 614 and $573 \mathrm{bp}$, respectively (Table 1). Barcode Pl-cd analysis of the four commercial products was successful in clearly identifying both rose hip- and hibiscus-specific fragments (Figure 5A), and no additional band was observed to suspect the presence of undeclared plant material in the samples. The barcode Pl-ef profile of the hibiscus reference had two bands of 260 and 469 $\mathrm{bp}$, whereas the rose hip reference produced a single $472 \mathrm{bp}$ fragment (Table 1). When the commercial products were analyzed, the hibiscus-specific $260 \mathrm{bp}$ fragment was readily identifiable in all four samples. However, the second, larger fragment could not be resolved by the capillary electrophoresis system in any of the replicate experiments because of the size similarity between the rose hip- and hibiscus-specific fragments (472 vs $469 \mathrm{bp}$ ) (Figure 5B, ,panels III-VI). As a result, whereas the presence of hibiscus in the samples was apparent by the presence of the $260 \mathrm{bp}$ fragment, the mixture of the 472 and $469 \mathrm{bp}$ fragments was identified as a single band within a size range of 470-472 bp. Nevertheless, barcode Pl-cd alone allowed distinguishing of species-specific amplification profiles when tested on rose hip-hibiscus blends.

Two types of fennel tea blends (anise-fennel tea and peppermint-fennel tea) were included in our analyses. Commercial samples of anise-fennel blends were labeled as either "fennel tea" or "anise-fennel tea" but always included anise in the list of ingredients. The ratio of anise in the blend was declared on the label of one product (sample 2) as $10 \%$,

\section{Rose hip Tea}

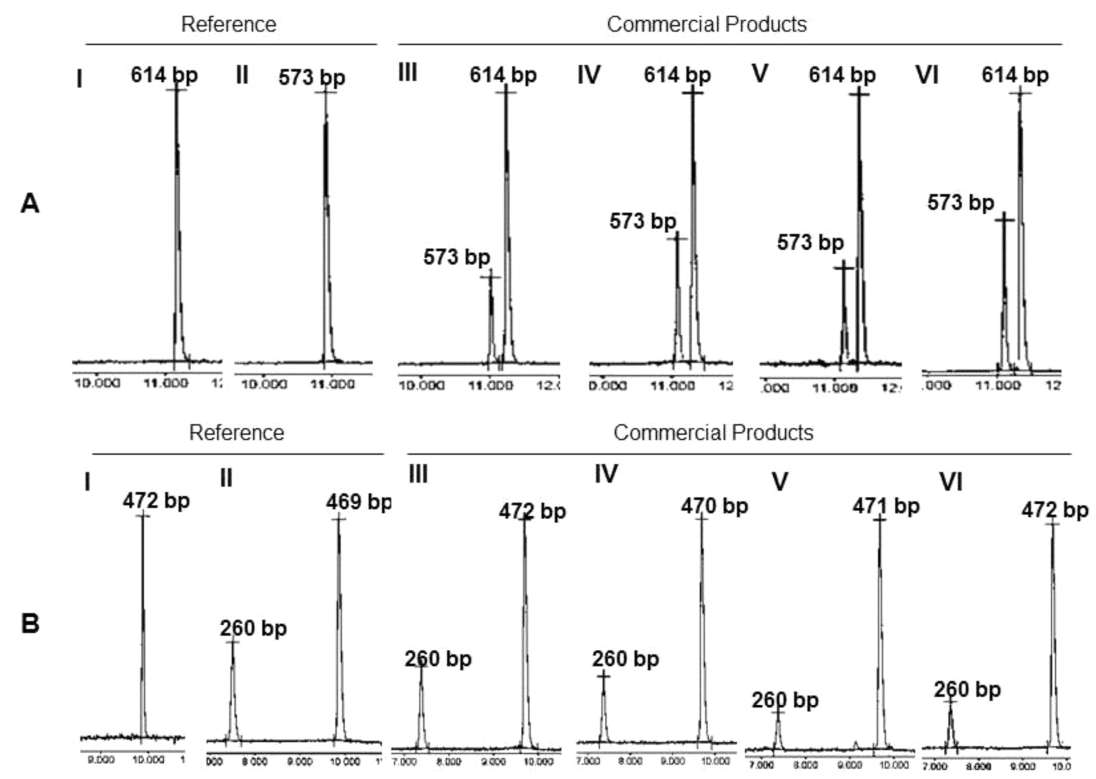

Figure 5. Capillary electropherograms displaying the results of the PCR-CE barcode assays applied on rose hip tea samples: (A) barcode Pl-cd assay; (B) barcode Pl-ef assay. Sample order is the same for rows A and B: I, rose hip reference sample; II, hibiscus reference sample; III, rose hip tea sample 1; IV, rose hip tea sample 2; V, rose hip tea sample 3; VI, rose hip tea sample 4. 


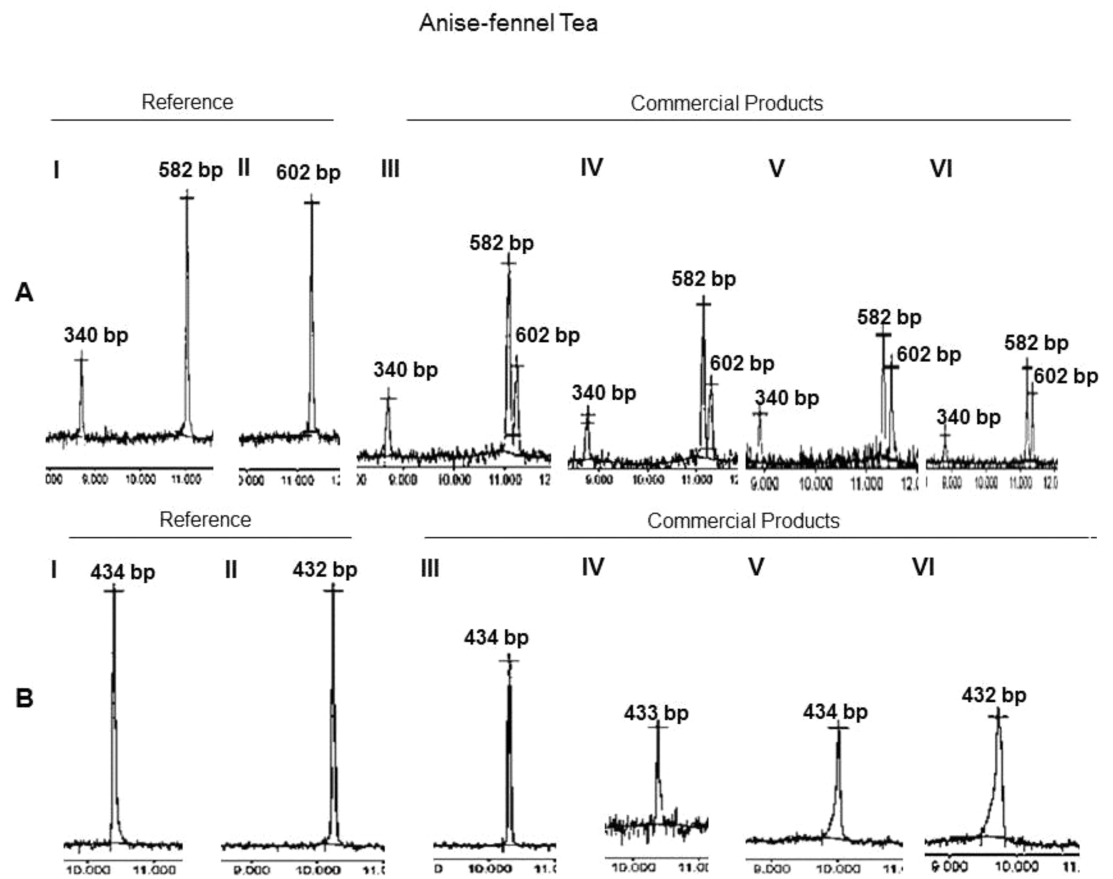

Figure 6. Capillary electropherograms displaying the results of the PCR-CE barcode assays applied on anise-fennel tea samples: (A) barcode Pl-cd assay; (B) barcode Pl-ef assay. Sample order is the same for rows A and B: I, fennel reference sample; II, anise reference sample; III, anise-fennel tea sample 1; IV, anise-fennel tea sample 2; V, anise-fennel tea sample 3; VI, anise-fennel tea sample 4.

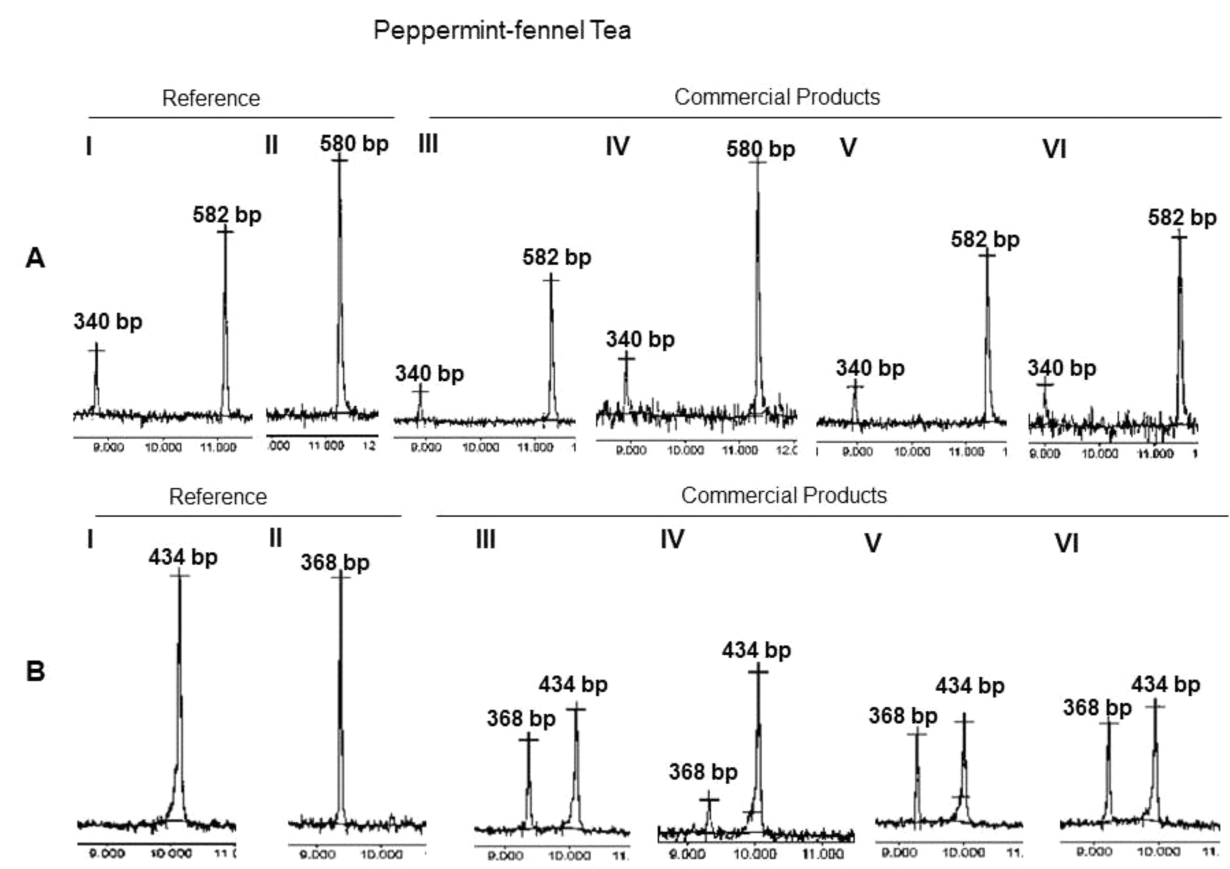

Figure 7. Capillary electropherograms displaying the results of the PCR-CE barcode assays applied on peppermint-fennel tea samples: (A) barcode Pl-cd assay; (B) barcode Pl-ef assay. Sample order is the same for rows A and B: I, fennel reference sample; II, peppermint reference sample; III, peppermint-fennel tea sample 1; IV, peppermint-fennel tea sample 2; V, peppermint-fennel tea sample 3; VI, peppermint-fennel tea sample 4.

but no ratio information was included in the ingredients list for the remaining three samples. The barcode Pl-cd amplification profile of fennel reference sample produced two bands of 340 and $582 \mathrm{bp}$, and the anise reference yielded a single $602 \mathrm{bp}$ fragment (Table 1). All three fragments representing the two species were resolved by the capillary electrophoresis system, clearly displaying the presence of both species in the samples (Figure 6A). In addition, there were no unexpected amplicons in the profiles of the commercial samples to suspect contamination or adulteration. Fennel and anise references produced barcode Pl-ef amplicons of similar size (434 and 432 bp) (Table 1). Although we were able to reproducibly distinguish the two species from each other when amplicons from individual reference samples were run simultaneously, fennel- and anise-specific barcode Pl-ef fragments could not be resolved when amplified from the tea blends. Such samples had 


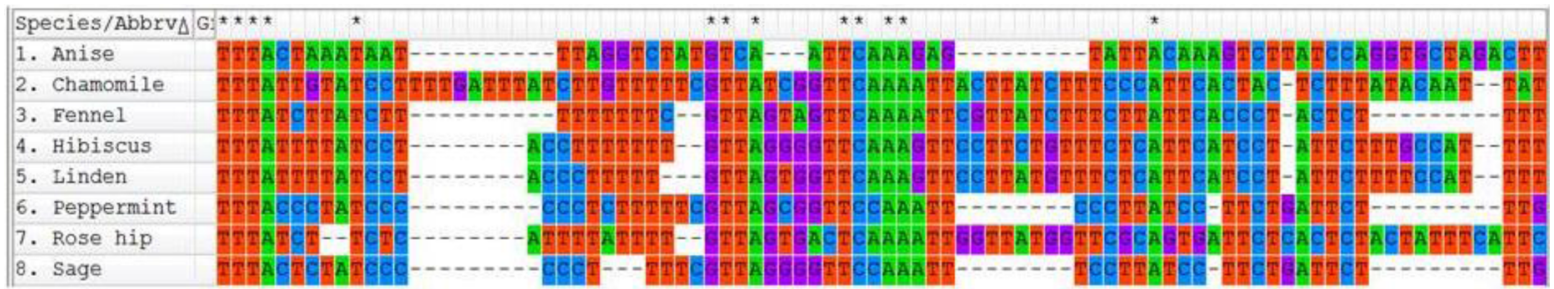

Figure 8. Portion of the alignment of plastid intergenic spacer ( $\operatorname{trnL}(\mathrm{UAA}) 3^{\prime}$ exon-trnF (GAA) gene) nucleotide sequences displaying indels and substitutions among eight herbal species.

single fragments of 432, 433, or 434 bp (Figure 6B, panels III$\mathrm{VI})$. Hence, barcode Pl-cd analysis was required to correctly identify the fennel-anise tea blends. For peppermint-fennel teas, blend ratio information was supplied on the labels of samples 1 and 2 as $90 \%$ fennel:10\% peppermint. Barcode Pl-cd and Pl-ef amplicon sizes of the peppermint reference sample were 580 and $368 \mathrm{bp}$, respectively (Table 1). Because the 582 $\mathrm{bp}$ fragment from fennel and $580 \mathrm{bp}$ fragment from peppermint could not be resolved, a two-fragment pattern with the $340 \mathrm{bp}$ fennel-specific band and a larger band of either 580 (Figure 7A, panel IV) or $582 \mathrm{bp}$ (Figure 7A, panels III, V, and VI) was observed for the peppermint-fennel tea samples as a result of barcode Pl-cd analysis. On the other hand, barcode Pl-ef was successful in clearly identifying the species-specific fragments of $434 \mathrm{bp}$ (fennel) and $368 \mathrm{bp}$ (peppermint) (Figure 7B). No unexpected barcode amplicon was observed in any of the replicate experiments to suspect the authenticity of the peppermint-fennel tea samples.

Sequencing Barcode Amplicons from Reference and Commercial Herbal Tea Samples. The two noncoding plastid barcodes used in this work represent single-copy sequences, as they are intended for use in plant phylogenetic inference. $^{21}$ Therefore, amplification profiles of both barcodes should have single fragments, and additional bands of lower intensity are regarded as nonspecific. Because the barcode Pl-ef amplification profile of hibiscus involved two fragments, only the larger fragment (469 bp), which was of comparable size with those from the remaining herbal species, was isolated from agarose gel for sequencing. Similarly, Spaniolas et al. ${ }^{24}$ reported extra fragments in the trnL (UAA) intron amplification profiles of soybean (Glycine max) and corn (Zea mays), which they regarded as unspecific PCR byproducts. Alignment of barcode Pl-ef sequences from reference samples revealed several insertions/deletions of variable size among the sequences from the eight species, which resulted in the length polymorphisms among the barcode amplicons (Figure 8).

To verify the sequence identity between barcode amplicons from the reference samples and the commercial products, barcode fragments from the commercial tea samples were also sequenced. For the three single-ingredient herbal teas (sage, linden, and chamomile), barcode-Pl-ef sequences from the tea samples perfectly matched those from the references (data not shown). To identify the contaminant/adulterant species in chamomile tea sample 4, the extra fragments that appeared in the barcode Pl-cd (Figure 2A, panel V) and Pl-ef (Figure 2B, panel V) profiles of the sample were isolated from agarose gel and sequenced. Genbank database search identified the highest identity scores for homologous sequences from the Rosmarinus officinalis (rosemary) plastid genome for both barcodes. According to the BLAST homology search results, barcode $\mathrm{Pl}$-cd sequence from the contaminant/adulterant species displayed an identity score of $96 \%$ with the rosemary sequence deposited under GenBank accession no. AY540465.1. Similarly, a 99\% identity score was displayed for the barcode Pl-ef sequence and a rosemary plastid sequence entry (GenBank accession no. FJ490792.1). As a result, we predicted that rosemary was the source of the unexpected barcode fragments in the chamomile tea. Barcode Pl-ef PCR products from the commercial peppermint-fennel and peppermint-linden tea samples were separated by agarose gel electrophoresis to isolate species-specific fragments separately for sequencing. In concordance with the results of the PCR-CE barcode assays, sequences obtained from the commercial samples exactly matched the peppermint, fennel, and linden reference barcode sequences. Because barcode Pl-ef amplicons of rose hip and hibiscus (Figure 5B) and fennel and anise (Figure 6B) could not be resolved due to their size similarity, species-specific fragments of barcode $\mathrm{Pl}-\mathrm{cd}$ were isolated and sequenced for the rose hip-hibiscus and anise-fennel commercial tea samples. Alignment of the sequence electropherograms showed that barcode sequences from the commercial tea samples were identical with the reference sequences for all four herbal species, thereby validating the results of the PCR-CE barcode analyses.

Determining the Threshold of Admixture Detection. To test the efficiency of the proposed methodology to identify blends of more than two herbal species and set a threshold of admixture detection, barcode Pl-cd assay was applied on inhouse blends prepared using reference herbal samples (Figures 9 and 10). To improve the confidence in assessing the performance of the proposed methodology, experiments were carried out using two different types of DNA polymerases. The two admixtures prepared with tissue from three herbal species contained $33 \%$ of each species in the blends. The barcode Pl-cd assay was successful in identifying the botanical origin of the two blends as admixtures of sage, anise, and chamomile (Figure

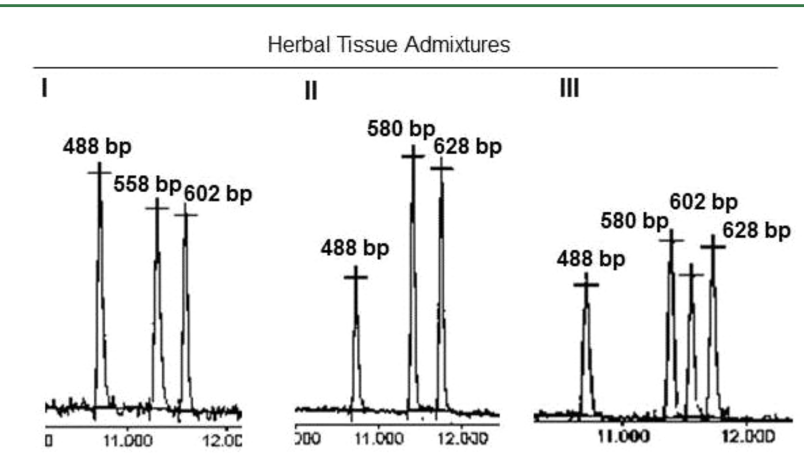

Figure 9. Capillary electropherograms displaying the results of the barcode Pl-cd assay applied on reference tissue admixtures of three and four herbal species. I, chamomile, sage, anise; II, chamomile, peppermint, linden; III, chamomile, peppermint, anise, linden. 


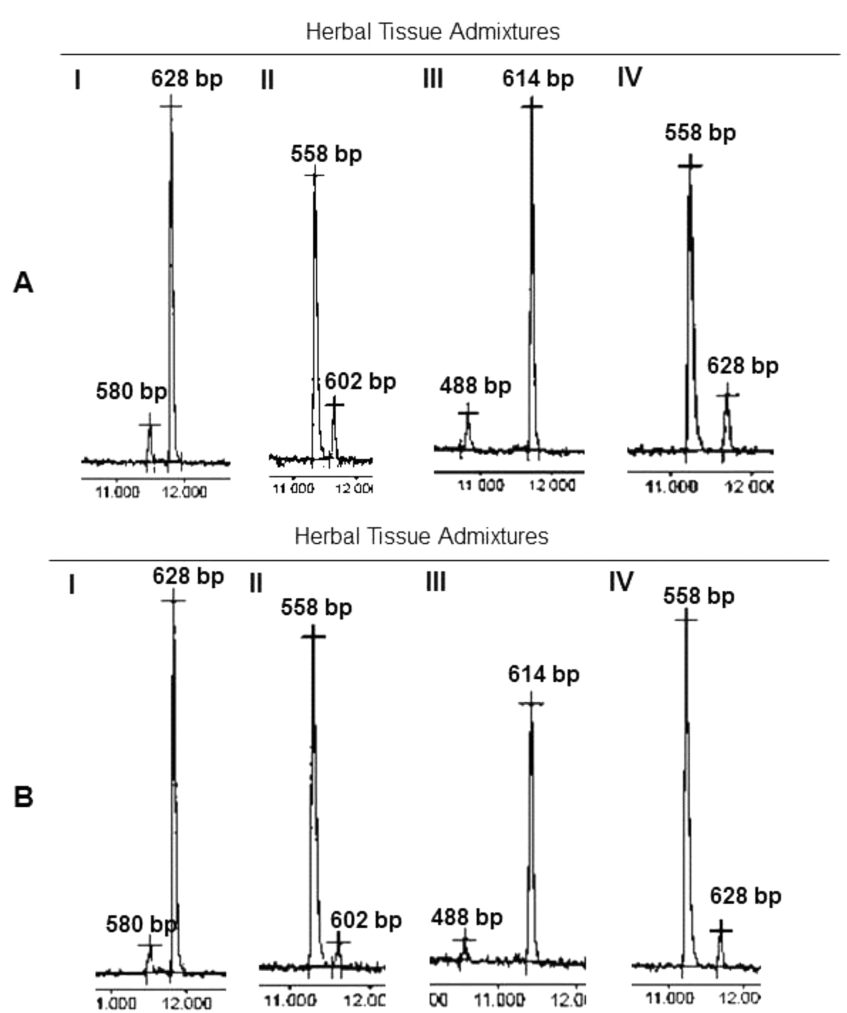

Figure 10. Capillary electropherograms displaying the results of the barcode Pl-cd assay applied on reference tissue admixtures of two herbal species: (A) 95:5\% blends; (B) 99:1\% blends. (A) I, 95\% linden:5\% peppermint; II, 95\% sage:5\% anise; III, 95\% rose hip:5\% chamomile; IV, 95\% sage:5\% linden. (B) I, 99\% linden:1\% peppermint; II, $99 \%$ sage:1\% anise; III, $99 \%$ rose hip:1\% chamomile; IV, $99 \%$ sage: $1 \%$ linden.

9, panel I) and linden, peppermint, and chamomile (Figure 9, panel II). The admixture of four herbal species contained $25 \%$ of each species in the blend. When the barcode Pl-cd assay was applied on the admixture, barcode fragments of all four species (chamomile, peppermint, anise, and linden) were successfully resolved. Thus, the sample was correctly identified as a blend of four different species (Figure 9, panel III). Linden:peppermint, sage:anise, rose hip:chamomile, and sage:linden admixtures were prepared in two sets that contained the minor constituent at ratios of 5 and $1 \%$ in the blends. When the barcode Pl-cd assay was applied on these admixtures, barcode amplicons from the minor constituents (peppermint, anise, chamomile, and linden, respectively) were readily identifiable in all of the samples for both admixing ratios (Figure 10). Parallel experiments with both DNA polymerases (GoTaq G2 Flexi DNA Polymerase, Promega Corp.; and Q5 High-Fidelity DNA Polymerase, New England Biolabs Inc.) produced similar results with similar barcode amplicon peak heights obtained from the minor constituents (data not shown). As a result, the threshold of admixture detection of the proposed methodology was set as $1 \%$.

\section{DISCUSSION}

It is almost impossible to identify substitution or contamination in herbal products because morphological features are lost during the processing of herbs into powders or fragmented products. ${ }^{2}$ Identification often requires sophisticated analytical chemistry methods or diagnostic molecular approaches. In addition, substitution can easily be masked with food colorants and aromatics, resulting in products with indistinguishable color, taste, and smell. As a result, herbal products constitute an easy target for fraud or mislabeling, as evidenced by recent DNA-based studies that report a high frequency of substitution in herbal teas ${ }^{1}$ and supplements. ${ }^{2,14,15}$

In this work, we tested the performance of plastid noncoding DNA length polymorphisms to authenticate the botanical origin of herbal teas. The plastid trnL (UAA) intron used in this work (barcode Pl-cd) was reported as a region of significant size variation, when sequences flanked by primers $c$ and $d^{21}$ were compared for 706 plant species corresponding to 366 genera and 116 families. ${ }^{16}$ Similarly, interspecific length polymorphisms that allowed species discrimination even within the same genus were shown for the plastid intergenic spacer [trnL (UAA) 3' exon-trnF (GAA) gene], ${ }^{21}$ which was used as the second barcode (barcode Pl-ef) in our study. By taking advantage of the length polymorphisms in the two noncoding barcode regions, we were able to verify the botanical origin and detect the presence of undeclared plant material in the tested herbal tea samples. For our sample set, the lowest ratio of an ingredient $(10 \%)$ was declared for anise and peppermint in anise-fennel and peppermint-fennel blends. Our results showed that we could clearly detect the presence of a minor ingredient in a commercial sample with the PCR-CE assays (Figures 6A, panel IV, and 7B, panels III and IV). When the barcode Pl-cd assay was applied on herbal tissue admixtures of more than two species, all herbal constituents in the blends were correctly identified. Moreover, herbal ingredients present in minor quantities as low as $1 \%$ were readily detected in the tested blends. In addition, two different DNA polymerases were equally efficient in amplifying the barcode fragment from minor constituents to detectable quantities. As a result, the limit of the proposed methodology to identify herbal tissue admixtures was identified as $1 \%$. We attribute this result to our barcode choice. Due to its high copy number in plant cells, the plastid genome is an ideal target for working with mixed samples of multiple species origin or highly processed samples with degraded DNA. $^{16}$

Following a similar PCR-CE approach, Spaniolas et al. ${ }^{24,25}$ used trnL (UAA) intron length polymorphisms for the authentication of the botanical origin of plant oils. While they were able to obtain barcode fragments of identical size as the references when they applied the PCR-CE assay on DNA from single plant species, amplicons with small size differences could not be resolved by the capillary electrophoresis system when PCR fragments from multiple species were analyzed. We also observed the problem of resolving small fragment size differences of 2-3 bp with barcode Pl-ef (Figure 5) and barcode Pl-cd (Figure 7A), when barcode amplicons from tea blends were analyzed. However, the use of a second barcode solved this problem in all three cases (Figures 5A, 6A, and 7B), resulting in the successful identification of herbal species in the tea blends. Thus, our results highlight the importance of using a combination of barcode regions to achieve sufficient species resolution. The use of barcode combinations is also valid when sample genotyping is based on sequencing. For example, Stoeckle et al. ${ }^{1}$ investigated the authenticity of commercial herbal teas based on $r b c L$ and $m a t K$ sequences and reported that readable sequences could be obtained from 77 and $79 \%$ of the samples when $r b c L$ and $m a t K$ regions were sequenced, respectively. However, the success rate for sequencing either of the markers was much higher (90\%). In our study, we 
established reference barcode fragment length profiles and reference barcode sequences using reference tissue samples of the eight herbal species. We assessed the authenticity of the commercial samples by running reference and sample barcode amplicons simultaneously on the capillary electrophoresis. Thus, we were able to compare the electrophoretic mobility of the barcode amplicons from samples and references with high confidence. In addition, we verified our PCR-CE analysis results by comparing the reference and sample barcode sequences. Because we performed reference sequencing in our study, we did not face the problem of finding barcode sequences from the relevant species in public databases. When a reference sample set is not employed, the success of identifying the botanical origin of a sample depends on the availability of sequence for the species in the databases. For example, Stoeckle et al. ${ }^{1}$ reported that barcode sequence records were missing in the GenBank database for about onethird of the herbal species listed on the labels of the tea samples they examined. In addition, it should be noted that, even when a sequence match is identified from a database, there is no certainty that the sequence entry is from a correctly identified plant. $^{2}$

In this work, we used plastid DNA length polymorphisms for the authentication of botanical origin in herbal teas. While species identification based on barcode sequencing works well with single-ingredient samples, sequencing reactions may fail to produce clean, reliable sequences from samples of multiple species due to sequence divergence. ${ }^{17}$ In that respect, barcode size variations improve the success rate in sequencing by enabling fragment isolation from a mixture of amplicons. By taking advantage of mitochondrial DNA length polymorphisms, Pun et al. ${ }^{30}$ proposed a methodology that involved PCR fragment separation followed by sequencing for the identification of species from mixed samples of unknown identity. Barcode fragment size-based tests are especially suitable for food analyses, because species origin authentication of food products aims at identifying whether a sample is true-to-label or not. Therefore, it is feasible to set standards by analyzing sample barcode fragments with respect to barcode profiles of ingredients listed on the product label. Any deviation from the standards (i.e., reference profiles of the listed ingredients) can be further investigated by taking advantage of barcode length polymorphisms to separate and isolate the alien fragment for sequencing. By following such an approach, we were able to isolate and sequence contaminant/adulterant barcode amplicons from the suspected chamomile tea sample and search public databases for homologous sequence matches. On the basis of our results, we predicted that chamomile tea sample 4 contained rosemary and was mislabeled as a single-ingredient product.

It is important to note that the utility of the PCR-CE barcode assays proposed in this work is not limited to herbal teas but can be extended to authenticate all types of processed herbal products or raw material. When an appropriate genomic region with sufficient interspecific size variation is selected as the analyte, capillary electrophoresis analysis is a faster, more costefficient, less equipment and labor/expertise demanding, and relatively simple alternative to sequencing for herbal product authenticity analyses. PCR-CE assays can be standardized for individual products by employing references based on the ingredients list and applied as routine tests for the verification of botanical origin. PCR-CE assays can also be coupled with sequencing any time adulteration is suspected in a sample.

\section{AUTHOR INFORMATION}

\section{Corresponding Author}

*(S.D.) E-mail: samidoganlar@iyte.edu.tr. Phone: +90 232750 7313.

\section{Author Contributions}

"A.T.U. and A.O.U. contributed equally to this paper.

Notes

The authors declare no competing financial interest.

\section{ACKNOWLEDGMENTS}

We are grateful to Aegean University Botanical Garden and Herbarium Research and Application Center (Izmir, Turkey) for providing reference herbal material.

\section{REFERENCES}

(1) Stoeckle, M. Y.; Gamble, C. C.; Kirpekar, R.; Young, G.; Ahmed, S.; Little, D. P. Commercial teas highlight plant DNA barcode identification successes and obstacles. Sci. Rep. 2011, 1, 42.

(2) Newmaster, S. G.; Grguric, M.; Shanmughanandhan, D.; Ramalingam, S.; Ragupathy, S. DNA barcoding detects contamination and substitution in North American herbal products. BMC Med. 2013, 11, 222.

(3) Ekor, M. The growing use of herbal medicines: issues relating to adverse reactions and challenges in monitoring safety. Front. Pharmacol. 2014, 4, 177.

(4) Galimberti, A.; De Mattia, F.; Losa, A.; Bruni, I.; Federici, S.; Casiraghi, M.; Martellos, S.; Labra, M. DNA barcoding as a new tool for food traceability. Food Res. Int. 2013, 50, 55-63.

(5) Madesis, P.; Ganopoulos, I.; Sakaridis, I.; Argiriou, A.; Tsaftaris, A. Advances of DNA-based methods for tracing the botanical origin of food products. Food Res. Int. 2014, 60, 163-172.

(6) Lockley, A. K.; Bardsley, R. G. DNA-based methods for food authentication. Trends Food Sci. Technol. 2000, 11, 67-77.

(7) Pafundo, S.; Gulli, M.; Marmiroli, N. SYBR®GreenERreal-time PCR to detect almond in traces in processed food. Food Chem. 2009, $116,811-815$.

(8) Costa, J.; Mafra, I.; Oliveira, M. B. P. P. High resolution melting analysis as a new approach to detect almond DNA encoding for Pru du 5 allergen in foods. Food Chem. 2012, 133, 1062-1069.

(9) Madesis, P.; Ganopoulos, I.; Bosmali, I.; Tsaftaris, A. Barcode high resolution melting analysis for forensic uses in nuts: a case study on allergenic hazelnuts (Corylus avellana). Food Res. Int. 2013, 50, 351-360.

(10) Mayer, F.; Haase, I.; Graubner, A.; Heising, F.; Paschke-Kratzin, A.; Fischer, M. Use of polymorphisms in the $\gamma$-gliadin gene of spelt and wheat as a tool for authenticity control. J. Agric. Food Chem. 2012, $60,1350-1357$

(11) Sonnante, G.; Montemurro, C.; Morgese, A.; Sabetta, W.; Blanco, A.; Pasqualone, A. DNA microsatellite region for a reliable quantification of soft wheat adulteration in durum wheat-based foodstuffs by real-time PCR. J. Agric. Food Chem. 2009, 57, 1019910204.

(12) Marieschi, M.; Torelli, A.; Bruni, R. Quality control of saffron (Crocus sativus L.): development of SCAR markers for the detection of plant adulterants used as bulking agents. J. Agric. Food Chem. 2012, 60, 10998-11004.

(13) Olivieri, C.; Marota, I.; Rollo, F.; Luciani, S. Tracking plant, fungal and bacterial DNA in honey specimens. J. Forensic Sci. 2012, 57, $222-227$.

(14) Baker, D. A.; Stevenson, D. W.; Little, D. P. DNA barcode identification of black cohosh herbal dietary supplements. J. AOAC Int. 2012, 95, 1023-1034.

(15) Wallace, L. J.; Boilard, S. M. A. L.; Eagle, S. H. C.; Spall, J. L.; Shokralla, S.; Hajibabaei, M. DNA barcodes for everyday life: routine authentication of natural health products. Food Res. Int. 2012, 49, $446-452$. 
(16) Taberlet, P.; Coissac, E.; Pompanon, F.; Gielly, L.; Miquel, C.; Valentini, A.; Vermat, T.; Corthier, G.; Brochmann, C.; Willerslev, E. Power and limitations of the chloroplastic trnL (UAA) intron for plant DNA barcoding. Nucleic Acids Res. 2007, 35, e14.

(17) Hollingsworth, P. M.; Graham, S. W.; Little, D. P. Choosing and using a plant DNA barcode. PLoS One 2011, 6, No. e19254.

(18) Dong, W.; Liu, J.; Yu, J.; Wang, L.; Zhou, S. Highly variable chloroplast markers for evaluating plant phylogeny at low taxonomic levels and for DNA barcoding. PLoS One 2012, 7, No. e35071.

(19) Chase, M. W.; Cowan, S.; Hollingsworth, P. M.; van den Berg, C.; Madrinan, S.; Petersen, G.; Seberg, O.; Jorgsensen, T.; Cameron, K. M.; Carine, M.; Niklas, P.; Hedderson, T. A. J.; Conrad, F.; Salazar, G. A.; Richardson, J. E.; Hollingsworth, M. L.; Barraclough, T. G.; Kelly, L.; Wilkinson, M. A proposal for a standardized protocol to barcode all land plants. Taxon 2007, 56, 295-299.

(20) CBOL Plant Working Group.. A DNA barcode for land plants. Proc. Natl. Acad. Sci. U.S.A. 2009, 106, 12794-12797.

(21) Taberlet, P.; Gielly, L.; Pautou, G.; Bouvet, J. Universal primers for amplification of three non-coding regions of chloroplast DNA. Plant Mol. Biol. 1991, 17, 1105-1109.

(22) Gielly, L.; Taberlet, P. The use of chloroplast DNA to resolve plant phylogenies: noncoding versus $r b c L$ sequences. Mol. Biol. Evol. 1994, 11, 769-777.

(23) James, D.; Schmidt, A. M. Use of an intron region of a chloroplast tRNA gene (trnL) as a target for PCR identification of specific food crops including sources of potential allergens. Food Res. Int. 2004, 37, 395-402.

(24) Spaniolas, S.; Bazakos, C.; Awad, M.; Kalaitzis, P. Exploitation of the chloroplast trnL (UAA) intron polymorphisms for the authentication of plant oils by means of a Lab-on-a-Chip capillary electrophoresis system. J. Agric. Food Chem. 2008, 56, 6886-6891.

(25) Spaniolas, S.; Bazakos, C.; Spano, T.; Zoghby, C.; Kalaitzis, P. The potential of plastid trnL (UAA) intron polymorphisms for the identification of the botanical origin of plant oils. Food Chem. 2010, $122,850-856$.

(26) Madesis, P.; Ganopoulos, I.; Argiriou, A.; Tsaftaris, A. The application of Bar-HRM (barcode DNA-high resolution melting) analysis for authenticity testing and quantitative detection of bean crops (Leguminosae) without prior DNA purification. Food Control 2012, 25, 576-582.

(27) Ganopoulos, I.; Madesis, P.; Darzentas, N.; Argiriou, A.; Tsaftaris, A. Barcode high resolution melting (Bar-HRM) analysis for detection and quantification of PDO "Fava Santorinis" (Lathyrus clymenum) adulterants. Food Chem. 2013, 133, 505-512.

(28) Faria, M. A.; Magalhães, A.; Nunes, M. E.; Oliveira, M. B. P. P. High resolution melting of $t r n \mathrm{~L}$ amplicons in fruit juices. Food Control 2013, 33, 136-141.

(29) Tamura, K.; Stecher, G.; Peterson, D.; Filipski, A.; Kumar, S. MEGA6: Molecular Evolutionary Genetics Analysis version 6. 0. Mol. Biol. Evol. 2013, 30, 2725-2729.

(30) Pun, K. M.; Albrecht, C.; Castella, V.; Fumagalli, L. Species identification in mammals from mixed biological samples based on mitochondrial DNA control region length polymorphism. Electrophoresis 2009, 30, 1008-1014. 